\title{
Selección de Resoluciones de especial interés del Tribunal Central de Recursos Contractuales en el año 2012
}

\section{RECURSOS Nº 040 Y 053/2012 - RESOLUCIÓN Nº68/2011 DE 14 DE MARZO DE 2012: RÉGIMEN CONTRACTUAL AGRUPACIONES DE INTERÉS ECONÓMICO}

“La Ley 12/1991, de 29 de abril regula las Agrupaciones de Interés Económico (AIE) de las que señala que tendrán personalidad jurídica y carácter mercantil, y por finalidad, la de facilitar el desarrollo o mejorar los resultados de la actividad de sus socios. El artículo $3^{\circ}$ señala que su objeto se limitará exclusivamente a una actividad económica auxiliar de la que desarrollen sus socios.

La adjudicataria alega en primer lugar, citando informes 7/92 y 45/02 de la Junta Consultiva de Contratación Administrativa del Estado, 15/2006 de la Junta Consultiva de Canarias, 1/2009 de la Junta Consultiva de Extremadura, así como diversas sentencias del Tribunal de Justicia de las Comunidades Europeas, que una Agrupación de Interés Económico puede contratar con el Sector Público, toda vez que tiene personalidad jurídica propia y carácter mercantil. No nos detendremos en esta cuestión por no ser objeto de controversia en el recurso que estamos analizando.

Analicemos la documentación presentada por la adjudicataria para acreditar su solvencia, y sus alegaciones y las del órgano de contratación sobre este punto, toda vez que el recurrente cuestiona que sea válida la clasificación que poseen los miembros de la agrupación de forma individual para acreditar la de la propia Agrupación.

La hoja-resumen del pliego de cláusulas administrativas particulares que rige la presente licitación exigía que los licitadores estuvieran clasificados en el grupo R, subgrupo 1, categoría $A$. La recurrente señala que la adjudicataria no posee tal clasificación, y que no es válida la aportada por sus miembros de forma individual; la adjudicataria, en cambio, y el órgano de contratación, consideran que sí que es posible acreditar la solvencia de la Agrupación con base en la de sus miembros.

Respecto a los medios de acreditar su solvencia, la adjudicataria se refiere al artículo 52 de la Ley 30/2007, de Contratos del Sector Público que señala que "para acreditar la solvencia necesaria para celebrar un contrato el empresario podrá basarse en la solvencia y medios de otras entidades, independientemente de la naturaleza jurídica de los vínculos que tenga con ellas, siempre que demuestre que, para la ejecución del contrato, dispone efectivamente de esos medios". 
El órgano de contratación, por su parte, considera que deben aplicarse a la Agrupación, por analogía, las normas específicas de las Uniones Temporales de Empresas y, concretamente, los artículos 59 y 67 del Texto Refundido de la Ley de Contratos del Sector Público, según los cuales, para valorar y apreciar la concurrencia de clasificación, respecto de los empresarios que concurran agrupados en UTE, se atenderá a las características acumuladas de cada uno de ellos.

Los informes de los distintos órganos consultivos y jurisdiccionales citados por la adjudicataria en apoyo de sus planteamientos coinciden, como ya se ha señalado, en la posibilidad que tiene una Agrupación de Interés Económico para contratar con el Sector Público, pero de ninguno de ellos cabe deducir que la Agrupación de Interés Económico se encuentre exenta del cumplimiento de los requisitos exigidos para participar en una licitación determinada. Así por ejemplo, el informe de la Junta Consultiva de Contratación Administrativa de la Administración del Estado de 27 de febrero de 1992, tras señalar que la Agrupación de Interés Económico es una figura encajable o con rasgos muy similares a la sociedad mercantil, indica que desde el punto de vista de la clasificación deberá ser tratada como una sociedad. Para resolver el problema de la clasificación, indica el informe, deberán aplicarse los criterios generales de la vigente legislación de contratos del Estado, y analizar los elementos personales, materiales y de experiencia de la propia Agrupación, "sin que puedan ser tomados en consideración los que, en su día, determinaron la clasificación individualizada de cada uno de ellos" El citado informe concluye que la clasificación de la Agrupación de Interés Económico ha de producirse con independencia de la clasificación reconocida, en su caso, a los integrantes de la misma.

La Junta Consultiva de Contratación Administrativa de la Junta de Extremadura, en informe de 3 de marzo de 2009 referido específicamente a la contratación con las Administraciones Públicas por parte de las Agrupaciones de Interés Económico, señala, citando el informe 7/92 de la Junta Consultiva de la Administración del Estado antes analizado, que en el caso de que una Agrupación de Interés Económico concurra como tal a una licitación Pública, "el problema de la clasificación deberá ser resuelto con arreglo a los criterios generales de la vigente legislación de Contratos del Estado".

Añade la Junta Consultiva de Extremadura que, conforme al artículo 56.3 de la Ley 30/2007 de Contratos del Sector Público, "En el supuesto de personas jurídicas pertenecientes a un grupo de sociedades, y a efectos de la valoración de su solvencia económica, financiera, técnica o profesional, se podrá tener en cuenta a las sociedades pertenecientes al grupo, siempre y cuando la persona jurídica en cuestión acredite que tendrá efectivamente a su disposición, durante el plazo a que se refiere el artículo 59.2, los medios de dichas sociedades necesarios para la ejecución de los contratos".

El citado artículo 59.2, por su parte, se refiere al plazo de vigencia y revisión de las clasificaciones y establece los plazos en que deberá justificarse el mantenimiento de la solvencia económico-financiera y la solvencia técnica y profesional para la con310 servación de la clasificación obtenida. 
El informe de la Junta Consultiva de Extremadura considera que, pese a no ser el supuesto de las AIE exactamente el de personas jurídicas pertenecientes al mismo grupo de sociedades, esta fórmula puede ser de aplicación para acreditar la solvencia siempre y cuando quede documentalmente acreditado que cuenta con la efectiva y plena disposición de los medios materiales y de solvencia económico-financiera de sus socios que resulten necesarios para la ejecución del contrato de que se trate.

Pero conviene darse cuenta de que ambos artículos, el 56 y el 59 de la Ley 30/2007 (que se corresponden con los artículos 67 y 70 del Texto Refundido de la Ley de Contratos del Sector Público, aprobado por Real Decreto Legislativo 3/2011) se refieren a criterios y condiciones para obtener la clasificación y para su mantenimiento, sin que en ningún momento se contemple exención alguna de contar con la clasificación necesaria para participar en las licitaciones que así lo exijan. Por lo que, en todo caso, la solvencia de las empresas del grupo se podría tomar en consideración cuando la Agrupación solicitase la clasificación, pero en ningún caso supone que no esté obligada a acreditar que dispone de dicha clasificación si la licitación en la que pretende participar así lo exige, como es el caso que nos ocupa.

En el mismo sentido se ha manifestado el Tribunal de Justicia de las Comunidades Europeas que, en diversas sentencias- asuntos C-389/92, C-5/97, C-176/98- expresa que la acreditación de la solvencia puede realizarse mediante la descripción de medios que no son propiedad del licitador sino que pertenecen a otras empresas con las que mantiene vínculos directos o indirectos, siempre que pruebe ante el órgano de contratación que dispone de manera efectiva de los mismos para ejecutar el contrato.

Tras los razonamientos expuestos, la Junta Consultiva de Extremadura concluye, sobre la contratación de las AIE, que "a pesar de las limitaciones que se derivan de su carácter auxiliar, ello no debe suponer necesariamente su automática exclusión del proceso de selección del contratista. Habrá que efectuar el análisis individualizado en cada caso para determinar o no su exclusión".

Este Tribunal hace suyos los planteamientos de la Junta Consultiva de Contratación Administrativa del Estado y de la de Extremadura, coincidentes con la doctrina del Tribunal de Justicia europeo, y considera que nada impide que una Agrupación de Interés Económico contrate con un órgano del Sector Público, pero tendrá que cumplir en cada caso los requisitos exigidos en el procedimiento de licitación en el que desee participar.

En el caso que nos ocupa, para acreditar la clasificación requerida en el pliego, aportó únicamente los certificados de clasificación de cada una de las empresas que forman la agrupación, por lo que entiende el Tribunal que no cumplió con las exigencias del pliego ni de la normativa de contratación del Sector Público. El punto 6 de la hoja-resumen del pliego de cláusulas administrativas particulares, que concreta el 
apartado IV.1 de dicho pliego, determina que los licitadores que deseen participar en la presente convocatoria deben estar clasificados en el grupo R, subgrupo 1, categoría A. Por tanto, cualquier empresa que quisiera acudir a la licitación debía contar con dicha clasificación.

Ciertamente la Ley de Contratos del Sector Público (actualmente, el TRLCSP aprobado por Real Decreto Legislativo 3/2011, de 14 de noviembre) contempla en su artículo 52 (artículo 63 del Texto Refundido) la posibilidad de integrar medios externos para acreditar la solvencia, para lo que exige que demuestre que cuenta con dichos medios para la ejecución del contrato en cuestión.

Pero el artículo 54 del mismo texto legal señala que para contratar con las Administraciones Públicas la ejecución de contratos de servicios de presupuesto igual o superior a $120.000 €$, será requisito indispensable que el empresario se encuentre debidamente clasificado. Por ello, en este caso, el punto 6 de la hoja-resumen del pliego de cláusulas administrativas particulares exigía que los licitadores dispusieran de la clasificación en el grupo R, subgrupo 1, categoría A, Y más allá de que no consta en el expediente que haya aportado ninguna documentación acreditativa de que las empresas que integran la Agrupación hubieran puesto sus medios a disposición de la ejecución del presente contrato, no presentó clasificación alguna de la propia Agrupación, limitándose a presentar las certificaciones de clasificación de cada una de las empresas que integran dicha Agrupación.

Hay que concluir, por tanto, que la adjudicataria no acreditó cumplir el requisito de clasificación exigido en el punto 6 de la hoja-resumen del pliego, por lo que procedía su exclusión.

Respecto a la aplicación a la Agrupación de Interés Económico, por analogía, de la normativa específica de las UTEs en materia de clasificación, el propio órgano de contratación que defiende tal posición, señala que existen diferencias importantes entre ambas figuras asociativas: el sistema de colaboración entre empresarios en la UTE tiene un tiempo cierto para el desarrollo de una obra, servicio o suministro, mientras que la AIE se constituye por tiempo indefinido; la UTE se crea para ejecuciones de obras, servicios o suministros concretos, mientras que la AIE existe al margen de las ejecuciones concretas. Nada dice el órgano de contratación respecto a una diferencia fundamental, cual es la de la responsabilidad de los socios que, en el caso de las AIE es subsidiaria de la de la Agrupación, conforme estipula el artículo $5^{\circ}$ de la Ley 12/1991, de 29 de abril, de Agrupaciones de Interés Económico.

Y así como el órgano de contratación, tras analizar tales diferencias, concluye que no existe fundamento para desvirtuar el acuerdo recurrido, este Tribunal entiende, por el contrario, que la AIE carecería de los medios para acreditar la solvencia exigida y que no es posible aplicar a la AIE, por analogía, las reglas previstas para las 
responden solidariamente de las obligaciones de la misma mientras que en la AIE la responsabilidad de los socios es subsidiaria de la de la Agrupación; en una UTE las empresas que la forman manifiestan expresamente, al concurrir a una licitación, que tienen intención de participar en la ejecución del contrato de que se trate, para lo cual aportan los medios de que disponen. Como se ha señalado antes, en la documentación remitida al Tribunal no consta que las empresas que componen hayan suscrito manifestación alguna al respecto".

\section{RECURSO Nº50/2012 - RESOLUCIÓN Nº76/2012 DE 28 DE MARZO DE 2012: LA CONCESIÓN DE BIENES DE DOMINICO PÚBLICO NO ES SUSCEPTIBLE DEL RECURSO ESPECIAL CONTRACTUAL}

"Mediante anuncio publicado en el Boletín Oficial del Estado con fecha 15 de febrero de 2012, la Autoridad Portuaria convocó licitación pública para la gestión mediante concesión administrativa de determinadas zonas de dominio público portuario y la incorporación a la superficie de explotación otorgada en concesión de una serie de infraestructuras, superestructuras, espacios e instalaciones.

Excluyendo los contratos subvencionados pues el caso examinado no es uno de ellos evidentemente, podemos indicar que la competencia del Tribunal se extiende de forma exclusiva a los contratos públicos nominados sujetos a regulación armonizada, así como a los contratos de servicios comprendidos en las categorías 17 a 27 del Anexo II de la Ley y a los de gestión de servicios públicos que reúnan las condiciones indicadas. Por consiguiente, sentado el hecho de que la Autoridad Portuaria como órgano de contratación se integra en la Administración General del Estado, para determinar si el supuesto contemplado en el presente recurso puede considerarse incluido entre los tipos que contempla el artículo que acabamos de transcribir y por tanto caen dentro de la esfera de la competencia de este Tribunal los recursos interpuestos en relación a él, será necesario que podamos atribuirle la naturaleza de alguno de los contratos mencionados.

Sin lugar a dudas, los dos únicos tipos dentro de los cuales podría ser incardinado, inicialmente, son la concesión de obras públicas y el contrato de gestión de servicios públicos, también en su modalidad de concesión administrativa.

La recurrente le atribuye la naturaleza jurídica de estos últimos mientras que el órgano de contratación considera que se trata de una concesión demanial regulada en los artículos 81 y siguientes del Real Decreto Legislativo 2/2011, de 5 de septiembre, por el que se aprueba el Texto Refundido de la Ley de Puertos del Estado y de la Marina Mercante.

Delimitar con toda claridad uno y otro concepto de concesión es fundamental para establecer si estamos o no ante un recurso interpuesto contra acto recurrible en esta vía. 
A tal fin, debemos definir la concesión de servicios, de conformidad con el artículo 8 de nuestra Ley de Contratos del Sector Público como aquélla "en cuya virtud una Administración Pública [...], encomienda a una persona, natural o jurídica, la gestión de un servicio cuya prestación ha sido asumida como propia de su competencia por la Administración [...] encomendante". Y con mayor precisión, siguiendo los artículos 275 y

276 de la misma como el contrato en cuya virtud una administración cede a un particular la gestión a su riesgo y ventura de alguno de los servicios de su competencia que sean susceptibles de explotación por particulares y que no implique ejercicio de la autoridad inherente a los poderes públicos.

Por su parte la concesión demanial, a tenor de la regulación que de la misma hacen nuestras leyes, principalmente la Ley 33/2003, de 3 de noviembre, del Patrimonio de las Administraciones Públicas, puede definirse como el título mediante el cual se habilita el uso especial o privativo de un bien de dominio público por un particular.

Se deduce claramente de lo anterior que la determinación de a cuál de estos conceptos responde la concesión objeto del proceso adjudicador cuya convocatoria y pliego de bases impugna la recurrente, exige un análisis del contenido de este último de forma especial en lo que respecta al objeto de la concesión. En tal sentido, el indicado pliego dispone en su base primera que "es objeto de este concurso la explotación, conforme se define en las presentes bases y en los planos anexos, mediante la oportuna concesión administrativa, de: Unas zonas de dominio público portuario con un total aproximado de $75.946,00 \mathrm{m2}$, conforme se detalla en los planos adjuntos, que comprenden $75.806,00 \mathrm{~m}^{2}$ de superficie de espejo de agua en explotación (en adelante, $E A E$ ), así como una superficie terrestre de 140,00 $\mathrm{m}^{2}$ y la incorporación a la superficie de explotación otorgada en concesión de una serie de infraestructuras, superestructuras, espacios e instalaciones, según detalle que a continuación se relaciona:". Sigue una relación de instalaciones existentes en la actualidad en el puerto.

La simple lectura de esta base pone de manifiesto que el objeto de la concesión está constituido por una superficie de agua y por un terreno que cuenta con una serie de instalaciones todas ellas integradas en el dominio marítimo portuario de conformidad con lo establecido en el artículo 67 de la ya mencionada Ley de Puertos del Estado y Marina Mercante en relación el artículo 4 de la Ley 22/1988, de 28 de julio, de Costas. Se cumple así uno de los requisitos esenciales para poder calificar la concesión como demanial, cual es el hecho de que su objeto esté constituido por terrenos de dominio público. No obstante, atendiendo a la expresión empleada en la base primera al definir el objeto de la concesión indicando que este lo constituye la explotación de unas determinadas zonas, pudiera entenderse que estamos ante una concesión de servicio público puesto que la propia definición de este contrato en la ley 30/2007, de 30 de octubre, que antes hemos transcrito habla precisamente de la explotación de un servicio como contrapartida de su prestación por el concesionario.

314 Sin embargo, falta en el supuesto que analizamos claramente la idea de la existencia 
de un servicio público de carácter predominante, siendo obvio que lo que se cede no es tanto la posibilidad de prestar un servicio público, cuanto la explotación de un determinado terreno calificado jurídicamente como demanio natural. Ello no resulta desvirtuado por el hecho de que el concesionario deba facilitar a los usuarios el suministro de agua y electricidad para las embarcaciones amarradas pues ello no supone sino una característica de la propia instalación que debe contar con los puntos de conexión correspondientes pero sin que el suministro de una u otra constituya propiamente un servicio prestado por el concesionario.

Segundo. Tampoco constituye una contradicción con la naturaleza demanial de la concesión que analizamos el hecho de que se prevea la posibilidad de realizar obras de acondicionamiento, mejora o ampliación de las instalaciones existentes, pues evidentemente éstas carecen de la entidad necesaria como para desvirtuar el carácter de la misma y convertirla en una concesión de obras. Está claro, y así se deduce de la base segunda del pliego que el objeto concesional lo constituyen las instalaciones actuales, sin perjuicio de que se prevea la posibilidad de mejorar o acondicionar algunas de ellas o, incluso, de ampliarlas llegado el caso. Resulta evidente, tal como se configura la concesión que el derecho a explotar los amarres no constituye la retribución del concesionario en contrapartida por la realización de obras, tal como exige el apartado 1 del artículo 7 de la Ley de Contratos del Sector Público.

Tercero. Por otra parte, si atendemos a la regulación de la Ley de Puertos del Estado y Marina Mercante, no ofrece duda que la concesión que analizamos debe ser calificada como de carácter demanial. En efecto, dicha Ley distingue entre concesiones demaniales y de obras, pero no regula, en relación con la explotación de los terrenos la figura de la concesión de servicios. Puesto que no se trata de una concesión de obras, según acabamos de ver, es obvio que la voluntad del legislador ha sido la de calificarla como concesión de dominio público, lo cual, por otra parte, es de toda lógica puesto que el objeto primordial de la explotación es un bien de dominio público.

En esta ocasión no cabe acudir a la reflexión que hicimos en nuestra resolución 154/2011 de 1 de junio del citado año, por la que resolvimos el recurso 116/2011, en relación con la necesidad existente, a efectos de determinar la competencia de este Tribunal, de calificar los contratos de conformidad con los criterios del derecho derivado de la Unión Europea y de las normas que lo incorporan a nuestro derecho, prescindiendo de las normas del derecho interno que les puedan atribuir una calificación jurídica diferente. Es evidente que ni la concesión de servicios ni la demanial son objeto de regulación por el derecho de la Unión Europea, por lo que la calificación adecuada en relación con una u otra debe hacerse exclusivamente a la luz de lo que dispongan nuestras leyes reguladoras de la materia.

Bien es cierto que este criterio no es de aplicación a la posible calificación de la concesión como de obras públicas, pues ésta si se encuentra regulada por el derecho de la Unión. Sin embargo, como acabamos de ver tal calificación no procede en el presente caso ni siquiera con arreglo a los requisitos exigidos por la legislación europea. 
Cuarto. Atendidos los razonamientos que preceden resulta claro que debemos declarar la falta de competencia del Tribunal para conocer del recurso interpuesto por no tratarse, la contemplada como objeto de la impugnación, de una concesión de obras ni de servicios, sino sobre bienes de dominio público y, por consiguiente, no incluida entre los supuestos en que se establece su competencia para resolver el recurso interpuesto contra los documentos contractuales que deben regir la adjudicación.

Asimismo debemos resolver sobre la solicitud formulada por la Autoridad Portuaria, como órgano de contratación interesado, en el sentido de que se revoque nuestra resolución acordando la suspensión provisional del expediente de contratación hasta que se resuelva el recurso. Tal resolución debería ser de inadmisión, pues como se desprende de lo dispuesto en el artículo 43.2, último párrafo, del Texto Refundido de la Ley de Contratos del Sector Público, contra los actos dictados en el procedimiento para la adopción de medidas cautelares no cabe recurso alguno. Sin embargo, dado que al dictar esta resolución acordamos el levantamiento de la misma de conformidad con lo que dispone el artículo 47.4 del texto refundido antes citado, la solicitud carece actualmente de fundamento, por lo que no es necesario hacer pronunciamiento expreso respecto de ella". 